\title{
Myocardial Cell Aging in the Elderly
}

\author{
Wenxi Li ${ }^{\mathrm{a}}$, Feng Liu ${ }^{\mathrm{a},{ }^{*}}$ \\ a Department of Geriatrics, Guangzhou First People's Hospital, School of Medicine, South China University of Technol- \\ ogy, Guangzhou 510180, Guangdong, China.
}

\begin{abstract}
Myocardial cell aging is closely related to cardiovascular disease. Stimulated by various factors, such as free fatty acid, glucose, hypoxia and radiation, cardiomyocyte aging involves a series of physiological and/or pathological changes. Cardiomyocytes are highly energy-consuming cells. Aging causes direct damage to cardiomyocytes, resulting in decreased myocardial contractility and reduced cardiac output, which affects the pumping function of the heart and eventually leads to heart failure. This article introduces the factors that influence myocardial cell aging in the elderly and the mechanisms responsible for these factors as well as provides suggestions for improving cardiovascular health and precise medicine of cardiovascular diseases in the elderly.
\end{abstract}

Keywords: Myocardial cell aging, influencing factors, related mechanisms

\section{Introduction}

Cardiovascular disease is a degenerative disease related to aging and it is the main disease leading to death and disability in the elderly. With the accelerated growth of the aging population, the incidence of cardiovascular diseases in the elderly is increasing. The high mortality rate, high hospitalization rate and high medical expenses of cardiovascular diseases add a heavy burden on families and society. Myocardial aging is closely associated with the occurrence of cardiovascular disease and is an important feature and independent risk factor of cardiovascular disease $[1,2]$. With aging, changes in genetic material, cell function, endocrine function, immunity, and metabolism take place in the body. These changes interact with and influence each other, leading to myocardial cell injury and apoptosis, and play an important role in the aging of myocardial cells. This article reviews the factors influencing myocardial cell aging its mechanisms of senescence in the elderly.

\section{Characteristics of myocardial aging and myo-}

\footnotetext{
* Corresponding author: Feng Liu

Mailing address: Department of Geriatrics, Guangzhou First People's Hospital, School of Medicine, South China University of Technology, Guangzhou 510180, Guangdong, China. Email: pfys1103@126.com
}

Received: 19 August 2020 / Accepted: 05 Septemper 2020

\section{cardial cell metabolism}

Myocardial aging is a kind of physiological and/or pathological change that affects the pumping function of the heart. Myocardial tissue is one of the most energy-consuming tissues in the body. Due to the great consumption of energy and oxygen in myocardial cells, mitochondria are abundant in them as mitochondria are the energy factories of cells. With aging, the ability of the body to heal from various kinds of stress-induced injuries decreases, causing dysfunction and a decreased number of mitochondria, in turn leading to the aging of myocardial cells. This aging causes a series of molecular changes in biology and cell metabolism, including changes of advanced glycation end products, PINK1/Parkin signaling pathway and mTOR signaling pathway, which manifest as a significant decrease in cell proliferation rate, increased apoptosis, arrest of the cell cycle, increased expression of aging markers ( $\beta$-galactosidase, tumor suppressor genes $\mathrm{p} 53$, $\mathrm{p} 16$, etc). These changes result in the homeostatic imbalance of cells, which causes the accumulation of damage to myocardial cells. This in turn results in decreases both in myocardial contractility and in cardiac output [3-5].

\section{Factors influencing myocardial cell aging and their mechanisms}

\section{Telomere dysfunction and DNA damage}

Telomeres play an important role in the process of human aging and diseases. Telomeres are located at the end of 
chromosomes and prevent cell aging and apoptosis [6]. In the process of cell replication, telomeres gradually shorten. This shortening limits cell division and proliferation, and eventually leads to DNA damage. When the shortening reaches a critical length, chromosomes lose their stability and mitochondrion dysfunction occurs. Cells no longer divide and become aged, which damages the repair function of tissues and organs, resulting in abnormal structures and functions in cells and tissues [7].

Van der Hart et al. [8] found that the telomere length of leukocytes in patients with congestive heart failure was shorter than that of the control group without the disease. Similarly, Brouilette et al. [9] found that the telomeres of leukocytes in the children of patients with coronary heart disease were shorter than those without coronary heart disease, and Collerton et al. [10] also confirmed in a clinical trial that the telomere lengths of peripheral blood leukocytes in elderly patients with heart failure were significantly shorter than those with normal heart function. Another study showed that telomeres in cardiomyocytes gradually shortened with aging; in adult animals, telomeres were shortened in 5-7\% of cardiomyocytes, while in the of elderly humans, telomere shortening occurred in $16 \%$ of cells [11]. Once telomeres are shortened to a certain length, cell cycle arrest is induced through the p53 gene pathway, which initiates cardiomyocyte apoptosis and then causes cardiomyocyte senescence. Therefore, telomere shortening is one of the biomarkers of aging.

Telomerase is the enzyme responsible for maintenance of the length of telomeres and it is an important sign of cell division. Increased and activated levels of telomerase can interfere with the process of cardiomyocyte division. For example, cardiomyocytes expressing telomerase have the ability to re-enter the cell proliferation cycle and perform multiplicative mitosis [12]. In mice, after the removal of telomerase, telomeres in cardiomyocytes were significantly shortened, and the expression of the p53 gene in the myocardium was up-regulated, leading to abnormalities in heart hemodynamics and heart anatomy [13].

Although some progress has been made in the research of telomeres, telomerase, and DNA damage, other details of the aging process and the molecular mechanisms related to them are still unclear. For instance,the key role in the process of telomere length maintenance with involvement of telomerase is still poorly studied. Furthermore, the expression of telomerase and the maintenance of telomere function are related to tumorigenesis. Therefore, caution is necessary in the development of interventional treatments involving telomeres, such as telomere shortening inhibition and the maintenance of telomerase activity [14]. Antiaging therapies related to telomeres and DNA damage still need further study and discussion.

\section{Oxidative stress and mitochondrial dysfunction}

Oxygen free radicals are continuously produced in the body, which relies on the free radical scavenging system to maintain the normal levels of these elements. With aging, and especially under the conditions of ischemia and hypoxia, the level of free radicals in the body increases, and there is an excess of reactive oxygen species (ROS). At the same time, the scavenging of oxygen radicals is reduced, resulting in the accumulation of free radicals in the human body. An excess of oxygen free radicals in the blood causes serious damage to cells, affecting proper cell formation and increasing the viscosity of cells. When passing through capillaries, cells are easily blocked due to the increase of their viscosity and form a micro embolism, which causes physiological dysfunction and induces various kinds of diseases associated with the elderly, including aging [15].

According to some research, oxidative stress not only directly damages cardiomyocytes but also leads to myocardial aging by causing mitochondrial dysfunction [16, 17]. Mitochondria play an important role in energy metabolism of cells by producing ATP. In cardiomyocytes, more than $90 \%$ of ATP is provided by mitochondria for the maintenance of myocardial homeostasis and contraction, making cardiomyocytes extremely sensitive to mitochondrial dysfunction $[18,19]$. ROS trigger apoptosis and senescence by opening the mitochondrial membrane permeability transition pore (mPTP) and by activating various enzymes and transcription factors to stimulate an inflammatory response $[20,21]$. The excess of ROS caused by oxidative stress also leads to damage of mitochondrial DNA (mtDNA) in cardiomyocytes, which directly causes abnormalities in mtDNA encoded peptides and results in protein dysfunction in the mitochondrial respiratory chain complex [22]. If this complex is defective, more free radicals are generated, accelerating mtDNA damage and causing cardiomyocyte aging and apoptosis [23].

Many studies have demonstrated mixed results regarding ROS and mitochondria.In a study with mice, mice with overexpression of mitochondrial catalase had significantly longer life spans than those without [24], demonstrating that mitochondrial catalase can reduce mitochondrial DNA damage caused by oxidative stress, and that it has a protective effect in myocardium. This effect includes an improvement of myocardial contractility and cardiac output [25]. However, recent studies have shown that ROS also have a positive effect on cardiomyocytes. A slight increase in ROS in the mitochondria of cardiomyocytes might activate the oxidative phosphorylation pathway and produce long-term gene protection. Song et al. [26] confirmed that ROS could induce autophagy of damaged mitochondria, indicating that a small amount of ROS may play a role in maintaining myocardial homeostasis.

In conclusion, oxidative stress and mitochondrial dysfunction are closely related to the aging of myocardial cells in the elderly. Therefore, early intervention of mitochondrial dysfunction and oxidative stress are important research targets in the clinical treatment of myocardial aging.

\section{Immunosenescence}

Immunosenescence is the gradual degradation of the immune system that occurs with the natural process of aging. As age increases, the immune response gradually decreas- 
es, and innate immunity and adaptive immunity degrade, causing disorders of the immune system and of cell function [27]. In the elderly, the process of immune system aging includes thymus degeneration, memory/effector $\mathrm{T}$ cell accumulation, depletion of initial $\mathrm{T}$ cells, decrease of $\mathrm{T}$ cell receptor polymorphism, and chronic inflammation [28-31]. In addition, aging of the immune system can also cause a decrease in the number of neutrophils and lymphocytes, a reduction in the effect of phagocytosis in polymorphonuclear cells, decreased production of superoxide, and a faster rate of apoptosis in peripheral blood mononuclear cells which participate in the restoration of hematopoietic and leukocyte cell lines [32]. The mechanism of immune system aging is complex, including gene recombination in cells and tissues, changes of cell differentiation and development, changes in signal regulation of cell proliferation, and apoptosis.

At present, there is no direct evidence to confirm the relationship between myocardial cell aging and the degradation of the immune system. But studies have shown that during myocardial infarction, neutrophils reach the obstruction site first, then other immunity cells such as monocytes and macrophages successively gather at the site of injury to participate in tissue repair [33]. In acute myocardial infarction, the SDF1-CXCR4 inflammatory axis between the injured myocardial tissue and the bone marrow, which favors the release of stem and mononuclear cells that participate in the replacement of cells of the leukocyte line during the acute phase of infarction [34]. Therefore, tissue repair may be affected when myocardial infarction occurs in older patients undergoing immunosenescence. Recent studies have found that an abnormal release of Neutrophil extracellular traps (NETs) may be related to aging. NETs are a kind of reticular structure formed by cytoplasmic proteins and nucleic acids that repair ruptures of the nuclear membrane. Reduced levels of NETs are related to atherosclerosis and myocardial infarction [35]. It is speculated that when myocardial infarction occurs in the elderly, the aging of the immune system affects the repair of vascular endothelial and leads to endothelial cell death, which may indirectly promote cardiomyocyte aging and death.

Autophagy is the fusion of cytoplasmic material and lysosomes through the formation of autophagic vesicles to induce the degradation of cytoplasmic proteins. Cellular autophagy plays an important role in resisting the adverse effects of heart aging by removing cytotoxic proteins and damaged organelles and by promoting the activation of the immune system [36, 37]. Many studies have shown that the decline of the autophagy function related to aging is associated with many cardiovascular diseases [38]. In a study conducted with older mice, Taneike et al. [39] found that the incidence of heart failure increased significantly in mice with a knockout gene for the specific autophagy effect on myocardium. They also showed that the left ventricular ejection fraction decreased significantly. Oyabu et al. [40] found that the decreased autophagy of cells could aggravate the occurrence of cardiac hypertrophy, one of the important morphological changes of myocardial aging. Therefore, the decline of autophagy may have a serious impact on the structure and function of myocardium by inducing aging. There are three types of autophagy, including microautophagy, chaperone-mediated autophagy (CMA), and macroautophagy [37]: In microautophagy, membrane invagination directly trapped and engulfed cytoplasmic cargo by lysosomes. CMA, on the other hand, directly transferred them to lysosomes and degraded cytosolic proteins with a KFERQ motif. In macroautophagy, small vesicular sacs called isolation membranes or phagophoresare initially formed, then the phagophores enclosed cytosolic long-lived proteins and organelles, resulting in the formation of double-membraned structures called autophagosomes, but the specific mechanism remains to be studied and confirmed.

\section{Chronic inflammation}

Aging is closely associated with the occurrence and development of chronic inflammation. With aging, the level of pro-inflammatory factors significantly increases, inducing cell senescence through oxidative stress, cell cycle arrest, and apoptosis. This in turn causes damage to multiple tissues, organs, and systems. C-reactive protein (CRP) is a non-specific molecular marker that reflects the systemic inflammatory response and is associated with many diseases, including cardiovascular diseases [41-43]. Harris et al. [44] conducted a 4.6-year follow-up observation on 1293 healthy elderly people and found a correlation between high CRP and mortality. Zhu et al. [45] found that the increase of CRP levels was related to an increased risk of accelerated aging and the prolongation of hospitalization. Walston et al. [46] found that the increase of CRP was related to frailty in the elderly after adjusting for basic demographic characteristics and excluding cardiovascular disease, diabetes, and other diseases.

Interleukin 6 (IL-6) is a pro-inflammatory cytokine also closely related to aging. High levels of IL-6 are found in elderly plasma, and its concentration is directly proportional to age. IL-6 is not only the main signal pathway regulating aging and cardiovascular disease but also a reliable aging biomarker and predictor of chronic inflammation [47]. Tumor necrosis factor (TNF-a) levels also increase with age, and imbalanced levels of TNF also play an important role in the process of cell aging [48]. Interleukin-10 (IL-10) is a cytokine with anti-inflammatory properties. Studies have shown that [49] once the function of IL-10 is altered, the balance of anti-inflammatory and pro-inflammatory responses will be upset, leading to increased levels of TNF-a and an inflammatory-aging reaction of tissues and organs. Lio et al. [49] found that when levels of IL-6 and TNF-a were increased, the levels of growth hormone and insulin-like growth factor (IGF-1) were down-regulated, which further confirmed the role of an inflammatory imbalance in promoting aging. Senescent cardiomyocytes secrete inflammatory cytokines, chemokines, growth factors, and proteases, known as the senescence-associated secretory (SASP). These changes affect 
the stability of the intracellular environment and cause further damage to cardiomyocytes, which manifests as left ventricular wall thickening, myocardial fibrosis, and decreased diastolic function, ultimately leading to changes in myocardial structure and function.

At present, more and more research has demonstrated that chronic inflammation and the aging of the immune system contribute to the process of aging, complementing and influencing each other $[50,51]$. They may share common cytokine receptors and molecular pathways and may regulate cell-cell and cell-cytokine interactions [52], known as the immunity-inflammatory-aging mechanism [47]. However, the relationship between chronic inflammation and immunosenescence and the pathological mechanism of this relationship are still unclear. As many morbid processes and diseases, including sarcopenia, dementia, osteoporosis and cancer, are characterized by chronic inflammation, which have shown a strong association with aging, it is significant for scientific and clinical researches to focus on this topic.

\section{Decreased estrogen}

Estrogens are steroid hormones, which are synthesized from cholesterol through a series of chemical reactions and have an important role in protecting the cardiovascular system and regulating metabolism. In the cardiovascular system, estrogen can protect the cardiovascular system through signaling pathways mediated by classical nuclear ER subtypes and membrane $G$ protein-coupled estrogen receptors (GPER) [53, 54]. Studies have shown that [55] estrogen can inhibit ventricular remodeling and left ventricular dilation by regulating the expression of extracellular matrix proteins.

The risk of cardiovascular diseases in elderly women is higher than that of elderly men, and the decrease in estrogen levels plays an important role. In elderly women, the decline of ovarian function leads to the reduced secretion of estrogen, accelerating the aging process and increasing the incidence of cardiovascular disease [56]. The lack of estrogen leads to increased ROS production in the body, thereby increasing endogenous oxidative stress damage and inducing changes in mitochondrial function as well as accelerating the process of apoptosis and promoting aging in the body [57]. Silencing regulator-related enzyme 1 (SIRT1) is closely associated with cell aging, genome stability, gene silencing, and energy metabolism [58]. Estrogen can up-regulate SIRTl in various ways to delay aging. The activation of SIRT1 can reduce oxidative stress damage and mitochondrial dysfunctionh [59], which are related to the pathological mechanism of cardiovascular disease [60]. The B lymphoma Mo-MLV insertion region 1 (Bmi-1) is a gene that is directly regulated by estrogen via estrogen receptor (ER) at the transcriptional level and it is one of the members of the Polycomb family of polythioprotein complexes [61]. The Bmi-1 gene can prevent cell aging though inhibiting transcription of the p16Ink4a/ p19Arf signaling pathway [62]. In addition, Bmi-1 can also prevent DNA damage and delay aging by maintaining mitochondrial function and redox balance [63]. Recent research has confirmed that estrogen deficiency can not only reduce the levels of antioxidant enzymes such as SOD1 and SOD2 in the mouse heart, but also reduce the expression of SIRT1 and Bmi-1, all these changes can increase oxidative stress in cardiomyocytes and promote senescence and apoptosis of cardiomyocytes in ovariectomized mice [64].

Although more and more studies have shown that estrogen reduction is related to myocardial aging in elderly women, the clinical therapy of estrogen replacement did not improve cardiac function in elderly women but increased the occurrence of cardiovascular events [65]. Therefore, there are still many unknown factors in the relationship between estrogen level and myocardial aging, and the mechanisms behind the relationship need to be further explored.

\section{Glucose metabolism disorders}

As the pancreas ages, endocrine function decreases and insulin sensitivity is reduced, causing disorders of glucose metabolism. At present, the relationship between glucose metabolism disorders and aging has not been confirmed in myocardial cells, but it has been confirmed in other types of cells. Song et al. [66] showed that the aging biomarkers of human endothelial progenitor cells were expressed under the stimulation of high glucose. In the development of diabetic cardiomyopathy, cardiomyocyte apoptosis is an important pathological change $[67,68]$ that is believed to cause the continuous loss of effective myocardial contractile units [69], promoting myocardial remodeling and ultimately leading to heart failure. Another important factor in cardiomyocyte apoptosis is oxidative stress. Persistent hyperglycemia induces oxidative stress and excessive ROS production. Yan et al. [70] reported that high glucose could participate in myocardial cell injury by regulating the Na+-K+ ATPase c-Src-dependent NADPH oxidase/ ROS pathway. Bian et al. [71] found that the P13K/AKT pathway mediated the protective effect of glucagon-like peptide (GLP-1) on high glucose-induced oxidative stress in rat cardiomyocytes, suggesting that the $\mathrm{P} 13 \mathrm{~K} / \mathrm{AKT}$ pathway is involved in high glucose stress myocardial cell injury in rats. Therefore, the disorder of glucose metabolism may promote the apoptosis of cardiomyocytes through oxidative stress, which leads to the aging of cardiomyocytes.

Glucose metabolism disorder can also lead to cardiomyocyte hypertrophy, which is an important phenotype of myocardial aging. Wang et al. [72] found that the Erk1/2 inhibitor had an inhibitory effect on rat cardiomyocyte hypertrophy induced by high glucose, indicating that the Erkl/2 pathway might mediate high glucose-induced rat cardiomyocyte hypertrophy. However, recent studies do not support the association between glycometabolism disorder and myocardial aging. Qian et al. [73] demonstrated that high glucose can damage the cardiac myocytes of rats by impairing their proliferation ability and reducing cellular survival, but that the high glucose environment did not increase the expression of myocardial cell aging bio- 
markers, suggesting that the damage of cardiac myocytes induced by high glucose did not cause cellular aging. At present, there is still a lack of research on glucose metabolism disorders and cardiomyocyte aging; more experiments are needed to verify the relationship between them.

\section{Disturbed lipid metabolism}

Free fatty acids (FFA) are important energy-supplying elements in cardiomyocytes. As the body ages, lipid metabolism begins to slow down, potentially leading to hyperlipidemia and abnormal lipid metabolism. FFA are closely related to cardiovascular disease in the elderly, and hyperlipidemia leads to cardiovascular events and mortality in this population $[74,75]$. Pilz et al. [76] conducted a follow-up study of 3315 patients over approximately 7 years and pointed out that FFA levels were independent risk factors for sudden cardiac death. More and more studies have shown that lipid metabolism disorder has become an independent predictor for sudden cardiac death, myocardial infarction, and cardiovascular mortality in the elderly $[76,77]$.

Too many FFA and their metabolites can cause lipid metabolism disorders and produce lipotoxicity [78]. Hyperlipidemia has an adverse effect on the myocardium, and the toxicity of lipotoxicity is much higher than that of glycotoxicity [79]. When fatty acid metabolism is disturbed, the uptake and utilization of fatty acids by cardiomyocytes are far greater than actual oxidative metabolism needs. A large amount of lipid accumulation in cardiomyocytes induces toxicity in the myocardium [80], causing damage to the cardiomyocytes and changing their structure and function [81]. An excess of FFA damages the membrane of cardiomyocytes, increases the production of ROS, interferes with the stability of ion channels on the membrane, and causes cardiomyocyte injury [82]. The myocardial toxicity caused by FFA hinders the release and utilization of glucose, reduces the production of ATP, and then leads to cardiac diastolic dysfunction, prolonged atrioventricular conduction, increased incidence of atrial fibrillation, and ultimately leads to heart failure [83].

A recent study showed that [65] under the stimulation of high FFA, human endothelial progenitor cells showed molecular evidence of aging, including increased expression of SA- $\beta$-gal, reduced angiogenesis, and decreased cell proliferation. Briot et al. found that excessive FFA induced increased expression of senescence biomarkers in human cardiac microvascular endothelial cells by inhibiting the cell cycle and up-regulating the expression of senescence-related inflammatory factors [84]. Palmitate (PA) is the largest component of FFA. PA can cause aging of human umbilical vein endothelial cells, and the increase of stimulation concentration and prolongation of action time increases the expression of SA- $\beta$-gal, apoptosis biomarkers, and levels of ROS, while the cell proliferation rate decreases and the normal cell cycle stagnates [85]. Sokolova found that PA can promote the expression of SASP in rat myocardial fibroblasts and can induce the expression of senescence-related biomarkers; the cell pro- liferation rate also decreases significantly [86]. Therefore, while PA can also induce changes in the aging phenotype in human cardiomyocytes, relevant research is still lacking, and the pathogenesis of myocardial aging related to lipid metabolism disorder has not been widely reported.

In summary, studying the correlation between lipid metabolism disorder and cardiomyocyte aging is expected to provide the basis for new interventions and treatments for cardiovascular disease in the elderly. Detailed study of the molecular mechanisms and potential targets of myocardial aging related to lipid metabolism disorder will play an important role in revealing its genetic basis, proteins, and cellular processes.

\section{Expectations}

As society ages, the concept of a full life cycle of health is becoming more and more popular. As the number one disease that seriously threatens the life and health of the elderly, cardiovascular disease has received more and more attention. Anti-aging has long been the focus of scientific research and clinicians. In particular, delaying heart aging and maintaining the pumping function of heart contraction and relaxation is the subject of unremitting efforts in the pursuit of clinical treatment of cardiovascular disease in the elderly.

However, the process of natural aging is inevitable. With the continuous improvement of the pursuit of the health of the elderly, the concept of "healthy aging" has attracted more and more attention from researchers. Research on human aging and reports on centenarians show that healthy aging is of great significance to maintaining the structure and function of myocardium. Therefore, it is important to discuss the characteristics and influencing factors of myocardial senescence in the elderly in order to improve cardiovascular health and regulate intervention and treatment measures of cardiovascular diseases.

However, myocardial cell senescence is a very complex process involving intricate molecular biological mechanisms and a large network of signal pathways. For example, advanced glycation end products might upregulate the level of mitochondrial autophagy in cardiomyocytes and cause cardiomyocyte senescence through their specific cell surface receptors and the homologous phosphatasetension protein-induced kinase (PINK1/Parkin) signaling pathway [87]. The rapamycin target protein (mTOR) signaling pathway transmits a variety of extracellular environmental signals. It regulates the physiological and pathological processes of the heart by generating adaptive responses in the cells, and the pathway plays an important role in cardiomyocyte aging [88]. The phosphatidylinositol kinase/protein kinase B (P13K/Akt) pathway can reduce oxidative stress and then delay peroxide-induced cardiomyocyte aging [89]. Similarly, it has been shown that the ubiquitin-mediated protein degradation system plays an important role in the proteasome degradation of cardiac proteins including myofibrillar proteins, con- 
nexin, and actin and myosin, and may have an effect on myocardial aging [90]. Finally, heat shock proteins (HSP) are proteins with highly conserved structures. HSP may delay myocardial aging through autophagy activation and mitochondrial activity improvement [91]. However, the research on the mechanism of myocardial aging is still incomplete, and there may be a common molecular mechanism among factors that influence myocardial aging in the elderly.

In summary, there are many factors related to myocardial aging in the elderly. Understanding these factors and analyzing their related signaling pathways, biological processes, and molecular mechanisms can provide a basis for the accurate treatment of cardiovascular disease in the elderly and further promote healthy aging, which is in turn expected to improve treatment effects, disease prognosis, and survival rate.

\section{Declarations}

Authors' contributions: Doctorial tutor Feng Liu conceived and designed the theme of the article. Student Wenxi Li collected information and finished the article.

Acknowledgements: The authors would like to thank Mr. Jose Luis Aceves for improving the English language of the manuscript.

Conflict of interest: The authors declare that they have no conflict of interest.

Consent for publication: This is an open-access article which permits unrestricted use, distribution and reproduction in any medium, provided the original authors and source are credited.

\section{References}

1. Lakatta E G, Levy D. Arterial and cardiac aging: major shareholders in cardiovascular disease enterprises: Part I: aging arteries: a "set up" for vascular disease. Circulation, 2003, 107(1): 139-146.

2. Najjar S S, Scuteri A, Lakatta E G. Arterial aging: is it an immutable cardiovascular risk factor? Hypertension, 2005, 46(3): 454-462.

3. Kida Y, Goligorsky M S. Sirtuins, cell senescence, and vascular aging. Canadian Journal of Cardiology, 2016, 32(5): 634-641.

4. Serrano M, Blasco M A. Putting the stress on senescence. Current opinion in cell biology, 2001, 13(6): 748-753.

5. Boon R A, Iekushi K, Lechner S, et al. MicroRNA-34a regulates cardiac ageing and function. Nature, 2013, 495(7439): 107-110.

6. Rizvi S, Raza S T, Mahdi F. Telomere length variations in aging and age-related diseases. Current aging science, 2014, 7(3): 161-167.

7. Zhu Y, Liu X, Ding X, et al. Telomere and its role in the ag- ing pathways: telomere shortening, cell senescence and mitochondria dysfunction. Biogerontology, 2019, 20(1): 1-16.

8. Van Der Harst P, van der Steege G, de Boer R A, et al. Telomere length of circulating leukocytes is decreased in patients with chronic heart failure. Journal of the American College of Cardiology, 2007, 49(13): 1459-1464.

9. Brouilette S W, Whittaker A, Stevens S E, et al. Telomere length is shorter in healthy offspring of subjects with coronary artery disease: support for the telomere hypothesis. Heart, 2008, 94(4): 422-425.

10. Collerton J, Martin-Ruiz C, Kenny A, et al. Telomere length is associated with left ventricular function in the oldest old: the Newcastle 85+ study. European heart journal, 2007, 28(2): 172-176.

11. Chang L S,Guo Z K. Research of Cardiac Telomerase. Advances In Cardiovascular Diseases.2005,26(5):547-550.

12. Leri A, Barlucchi L, Limana F, et al. Telomerase expression and activity are coupled with myocyte proliferation and preservation of telomeric length in the failing heart. Proceedings of the National Academy of Sciences, 2001, 98(15): 8626-8631.

13. Leri A, Franco S, Zacheo A, et al. Ablation of telomerase and telomere loss leads to cardiac dilatation and heart failure associated with p53 upregulation. The EMBO journal, 2003, 22(1): 131-139.

14. Sugarman E T, Zhang G, Shay J W. In perspective: An update on telomere targeting in cancer. Molecular carcinogenesis, 2019, 58(9): 1581-1588.

15. Harman D. The free radical theory of aging. Antioxidants and Redox Signaling, 2003, 5(5): 557-561.

16. Panth N, Paudel K R, Parajuli K. Reactive oxygen species: a key hallmark of cardiovascular disease. Advances in medicine, 2016, 2016.

17. Chaudhary K R, El-Sikhry H, Seubert J M. Mitochondria and the aging heart. Journal of geriatric cardiology: JGC, 2011, 8(3): 159.

18. Dongworth R K, Hall A R, Burke N, et al. Targeting mitochondria for cardioprotection: examining the benefit for patients. Future Cardiology, 2014, 10(2): 255-272.

19. Tocchi A, Quarles E K, Basisty N, et al. Mitochondrial dysfunction in cardiac aging. Biochimica et Biophysica Acta (BBA)-Bioenergetics, 2015, 1847(11): 1424-1433.

20. Bhatti J S, Bhatti G K, Reddy P H. Mitochondrial dysfunction and oxidative stress in metabolic disorders-A step towards mitochondria based therapeutic strategies. Biochimica et Biophysica Acta (BBA)-Molecular Basis of Disease, 2017, 1863(5): 1066-1077.

21. Verdin E. NAD+ in aging, metabolism, and neurodegeneration. Science, 2015, 350(6265): 1208-1213.

22. He $\mathrm{C}$, et al. Mitochondrial regulation of cardiac aging. Biochimica et Biophysica Acta (BBA)-Molecular Basis of Disease, 2019, 1865(7): 1853-1864.

23. Barja G, Herrero A. Oxidative damage to mitochondrial DNA is inversely related to maximum life span in the heart and brain of mammals. The FASEB Journal, 2000, 14(2): 312-318.

24. Kujoth G C, Hiona A, Pugh T D, et al. Mitochondrial DNA 
mutations, oxidative stress, and apoptosis in mammalian aging. Science, 2005, 309(5733): 481-484.

25. Dai D F, Santana L F, Vermulst M, et al. Overexpression of catalase targeted to mitochondria attenuates murine cardiac aging. Circulation, 2009, 119(21): 2789.

26. Song M, Chen Y, Gong G, et al. Super-suppression of mitochondrial reactive oxygen species signaling impairs compensatory autophagy in primary mitophagic cardiomyopathy. Circulation research, 2014, 115(3): 348-353.

27. Nikolich-Žugich J. The twilight of immunity: emerging concepts in aging of the immune system. Nature immunology, 2018, 19(1): 10-19.

28. Cepeda S, Griffith A V. Thymic stromal cells: Roles in atrophy and age-associated dysfunction of the thymus. Experimental gerontology, 2018, 105: 113-117.

29. Pawelec G. Does the human immune system ever really become "senescent"?. F1000Research, 2017, 6.

30. Linehan E, Fitzgerald D. Ageing and the immune system: focus on macrophages. European Journal of Microbiology and Immunology, 2015, 5(1): 14-24.

31. Shehata H M, Hoebe K, Chougnet C A. The aged nonhematopoietic environment impairs natural killer cell maturation and function. Aging Cell, 2015, 14(2): 191199.

32. Gonçalves M T, Mitchell T J, Lord J M. Immune ageing and susceptibility to Streptococcus pneumoniae. Biogerontology, 2016, 17(3): 449-465.

33. Nahrendorf M, Swirski F K, Aikawa E, et al. The healing myocardium sequentially mobilizes two monocyte subsets with divergent and complementary functions. The Journal of experimental medicine, 2007, 204(12): 30373047.

34. Anzai A, Anzai T, Nagai S, et al. Regulatory role of dendritic cells in postinfarction healing and left ventricular remodeling. Circulation, 2012, 125(10): 1234-1245.

35. Döring Y, Soehnlein O, Weber C. Neutrophil extracellular traps in atherosclerosis and atherothrombosis. Circulation research, 2017, 120(4): 736-743.

36. López-Otín C, Blasco M A, Partridge L, et al. The hallmarks of aging. Cell, 2013, 153(6): 1194-1217.

37. Shirakabe A, Ikeda Y, Sciarretta S, et al. Aging and autophagy in the heart. Circulation research, 2016, 118(10): 1563-1576.

38. Sasaki Y, Ikeda Y, Iwabayashi M, et al. The impact of autophagy on cardiovascular senescence and diseases. International Heart Journal, 2017, 58(5): 666-673.

39. Taneike M, Yamaguchi O, Nakai A, et al. Inhibition of autophagy in the heart induces age-related cardiomyopathy. Autophagy, 2010, 6(5): 600-606.

40. Oyabu J, Yamaguchi O, Hikoso S, et al. Autophagy-mediated degradation is necessary for regression of cardiac hypertrophy during ventricular unloading. Biochemical and biophysical research communications, 2013, 441(4): 787-792.

41. Cappola A R, Xue Q L, Ferrucci L, et al. Insulin-like growth factor I and interleukin- 6 contribute synergistically to disability and mortality in older women. The Journal of Clinical Endocrinology \& Metabolism, 2003,
88(5): 2019-2025.

42. Tsai A W, Cushman M, Rosamond W D, et al. Coagulation factors, inflammation markers, and venous thromboembolism: the longitudinal investigation of thromboembolism etiology (LITE). The American journal of medicine, 2002, 113(8): 636-642.

43. Eikelenboom P, Veerhuis R, Scheper W, et al. The significance of neuroinflammation in understanding Alzheimer's disease. Journal of neural transmission, 2006, 113(11): 1685.

44. Harris T B, Ferrucci L, Tracy R P, et al. Associations of elevated interleukin- 6 and C-reactive protein levels with mortality in the elderly. The American journal of medicine, 1999, 106(5): 506-512.

45. Zhu Y, Liu Z, Wang Y, et al. C-reactive protein, frailty and overnight hospital admission in elderly individuals: A population-based study. Archives of gerontology and geriatrics, 2016, 64: 1-5.

46. Walston J, McBurnie M A, Newman A, et al. Frailty and activation of the inflammation and coagulation systems with and without clinical comorbidities: results from the Cardiovascular Health Study. Archives of internal medicine, 2002, 162(20): 2333-2341.

47. Xia S, Zhang X, Zheng S, et al. An update on inflammaging: mechanisms, prevention, and treatment. Journal of Immunology Research, 2016, 2016.

48. Cesari M, Penninx B W J H, Pahor M, et al. Inflammatory markers and physical performance in older persons: the InCHIANTI study. The Journals of Gerontology Series A: Biological Sciences and Medical Sciences, 2004, 59(3): M242-M248.

49. Lio D, Scola L, Crivello A, et al. Inflammation, genetics, and longevity: further studies on the protective effects in men of IL-10- 1082 promoter SNP and its interaction with TNF- $\alpha-308$ promoter SNP. Journal of medical genetics, 2003, 40(4): 296-299.

50. Kramer G, Mitteregger D, Marberger M. Is benign prostatic hyperplasia (BPH) an immune inflammatory disease?European urology, 2007, 51(5): 1202-1216.

51. Macaulay R, Akbar A N, Henson S M. The role of the T cell in age-related inflammation. Age, 2013, 35(3): 563-572.

52. Nie K, Zhang Y, Gan R, et al. Polymorphisms in immune/ inflammatory cytokine genes are related to Parkinson's disease with cognitive impairment in the Han Chinese population. Neuroscience letters, 2013, 541: 111-115.

53. Meyer M R, Prossnitz E R, Barton M. The G protein-coupled estrogen receptor GPER/GPR30 as a regulator of cardiovascular function. Vascular pharmacology, 2011, 55(1-3): 17-25.

54. Prossnitz E R, Barton M. Estrogen biology: new insights into GPER function and clinical opportunities. Molecular and cellular endocrinology, 2014, 389(1-2): 71-83.

55. Mahmoodzadeh S, Dworatzek E, Fritschka S, et al. $17 \beta$-Estradiol inhibits matrix metalloproteinase- 2 transcription via MAP kinase in fibroblasts. Cardiovascular research, 2010, 85(4): 719-728.

56. Sitruk-Ware R. Estrogen, hormonal therapy and Aging. Bulletin de l'Academie nationale de medecine, 2015, 
199(2-3): 355-61; discussion 361-2.

57. Gupte A A, Pownall H J, Hamilton D J. Estrogen: an emerging regulator of insulin action and mitochondrial function. Journal of diabetes research, 2015, 2015.

58. Lv L, Shen Z, Zhang J, et al. Clinicopathologcal significance of SIRT1 expression in colorectal adenocarcinoma. Medical oncology, 2014, 31(6): 965.

59. Cheng Y, Di S, Fan C, et al. SIRT1 activation by pterostilbene attenuates the skeletal muscle oxidative stress injury and mitochondrial dysfunction induced by ischemia reperfusion injury. Apoptosis, 2016, 21(8): 905-916.

60. Ma L, Li Y. SIRT1: role in cardiovascular biology. Clinica chimica acta, 2015, 440: 8-15.

61. Li J, Wang Q Yang R, et al. BMI-1 Mediates Estrogen-Deficiency-Induced Bone Loss by Inhibiting Reactive Oxygen Species Accumulation and T Cell Activation. Journal of Bone and Mineral Research, 2017, 32(5): 962-973.

62. Mas-Bargues C, Viña-Almunia J, Inglés M, et al. Role of p16INK4a and BMI-1 in oxidative stress-induced premature senescence in human dental pulp stem cells. Redox biology, 2017, 12: 690-698.

63. Griffith J, Andrade D, Mehta M, et al. Silencing BMI1 radiosensitizes human breast cancer cells by inducing DNA damage and autophagy. Oncology Reports, 2017, 37(4): 2382-2390.

64. Yan Y, Hao Zhang H, Yang Z J, et al. Research of Estrogen Deficiency on Myocardial Aging, Apoptosis and Cardiac Function. Journal of Medical Research, 2018,47(12):110114.

65. Rodgers J L, Jones J, Bolleddu S I, et al. Cardiovascular risks associated with gender and aging. Journal of cardiovascular development and disease, 2019, 6(2): 19.

66. Song X, Yang B, Qiu F, et al. High glucose and free fatty acids induce endothelial progenitor cell senescence via PGC-1 $\alpha /$ SIRT1 signaling pathway. Cell Biology International, 2017, 41(10): 1146-1159.

67. Liu Z, Zhao N, Zhu H, et al. Circulating interleukin-1 $\beta$ promotes endoplasmic reticulum stress-induced myocytes apoptosis in diabetic cardiomyopathy via interleukin-1 receptor-associated kinase-2. Cardiovascular diabetology, 2015, 14(1): 1-9.

68. Filardi T, Ghinassi B, Di Baldassarre A, et al. Cardiomyopathy associated with diabetes: the central role of the cardiomyocyte. International journal of molecular sciences, 2019, 20(13): 3299.

69. Sun X, Chen R, Yang Z, et al. Taxifolin prevents diabetic cardiomyopathy in vivo and in vitro by inhibition of oxidative stress and cell apoptosis. Food and Chemical Toxicology, 2014, 63: 221-232.

70. Yan X, Xun M, Dou X, et al. Regulation of Na+-K+-ATPase effected high glucose-induced myocardial cell injury through c-Src dependent NADPH oxidase/ROS pathway. Experimental Cell Research, 2017, 357(2): 243-251.

71. Bian Y F, Wang D X, Yang H Y, et al. Glucagon like peptide-1 inhibits high glucose-induced injury of oxidative stress in cardiomyocytes of neonatal rats. Sheng li xue bao:[Acta physiologica Sinica], 2011, 63(4): 387-395.

72. WANG S, LIU Z, WANG L, et al. Effect of ERK1/2 inhibitor on cardiomyocyte hypertrophy induced by high glucose and insulin in neonatal rats. Journal of Shandong University (Health Sciences), 2007 (5): 05.

73. Wu Q, Li W J, He H, et al. Hyperglycemia Induces Cardiac Cell Apoptosis Via Regulating c-Src/PI3K/ERK Pathway. Journal of Hubei University of Science and Technology, 2019, 33(2):110-113.

74. Djoussé L, Benkeser D, Arnold A, et al. Plasma free fatty acids and risk of heart failure: the Cardiovascular Health Study. Circulation: Heart Failure, 2013, 6(5): 964-969.

75. Lv Z H, Ma P, Luo W, et al. Association between serum free fatty acid levels and possible related factors in patients with type 2 diabetes mellitus and acute myocardial infarction. BMC cardiovascular disorders, 2014, 14(1): 1-7.

76. Pilz S, Scharnagl H, Tiran B, et al. Elevated plasma free fatty acids predict sudden cardiac death: a 6.85-year follow-up of 3315 patients after coronary angiography. European heart journal, 2007, 28(22): 2763-2769.

77. Bhardwaj A, Truong Q A, Peacock W F, et al. A multicenter comparison of established and emerging cardiac biomarkers for the diagnostic evaluation of chest pain in the emergency department. American heart journal, 2011, 162(2): 276-282. e1.

78. Coort S L M, Bonen A, van der Vusse G J, et al. Cardiac substrate uptake and metabolism in obesity and type2 diabetes: role of sarcolemmal substrate transporters. Molecular and cellular biochemistry, 2007, 299(1-2): 5-18.

79. Chen D, Jiliang W U, Jing L I. Role of endoplasmic reticulum stress in high fattyacid induced injury in cardiomyocytes. Chinese Pharmacological Bulletin, 2017, 33(7): 966-971.

80. Li Q Yu Q, Na R, et al. Omega-3 polyunsaturated fatty acids prevent murine dilated cardiomyopathy by reducing oxidative stress and cardiomyocyte apoptosis. Experimental and therapeutic medicine, 2017, 14(6): 61526158.

81. Katunga L A, Gudimella P, Efird J T, et al. Obesity in a model of gpx4 haploinsufficiency uncovers a causal role for lipid-derived aldehydes in human metabolic disease and cardiomyopathy. Molecular metabolism, 2015, 4(6): 493-506.

82. Prihandoko R, Alvarez-Curto E, Hudson B D, et al. Distinct Phosphorylation Clusters Determine the Signaling Outcome of Free Fatty Acid Receptor 4/G ProteinCoupled Receptor 120. Molecular pharmacology, 2016, 89(5): 505-520.

83. Peterson J T, Li H, Dillon L, et al. Evolution of matrix metalloprotease and tissue inhibitor expression during heart failure progression in the infarcted rat. Cardiovascular research, 2000, 46(2): 307-315.

84. Briot A, Decaunes P, Volat F, et al. Senescence alters PPAR $\gamma$ (Peroxisome Proliferator-Activated Receptor Gamma)-dependent fatty acid handling in human adipose tissue microvascular endothelial cells and favors inflammation. Arteriosclerosis, thrombosis, and vascular biology, 2018, 38(5): 1134-1146. 
85. Li Y, Peng Z, Wang C, et al. Novel role of PKR in palmitateinduced Sirt1 inactivation and endothelial cell senescence. American Journal of Physiology-Heart and Circulatory Physiology, 2018, 315(3): H571-H580.

86. Sokolova M, Vinge L E, Alfsnes K, et al. Palmitate promotes inflammatory responses and cellular senescence in cardiac fibroblasts. Biochimica et Biophysica Acta (BBA)-Molecular and Cell Biology of Lipids, 2017, 1862(2): 234-245.

87. Zha Z, Wang J, Wang X, et al. Involvement of PINK1/Parkin-mediated mitophagy in AGE-induced cardiomyocyte aging. International journal of cardiology, 2017, 227: 201-208.

88. Johnson S C, Sangesland M, Kaeberlein M, et al. Modulat- ing mTOR in aging and health. Aging and Health-A Systems Biology Perspective. Karger Publishers, 2015, 40: 107-127.

89. Shen Y, Liu J X, Lei C,et al. Effect of Astragalosides on Oxidative Stress and PI3K/AKT Pathway in H2O2-induced Aging Cardiomyocytes. Journal of Guangzhou University of Traditional Chinese Medicine, 2019, 36(3):391-395.

90. Pagan J, Seto T, Pagano M, et al. Role of the ubiquitin proteasome system in the heart. Circulation research, 2013, 112(7): 1046-1058.

91. Lin S, Wang Y, Zhang X, et al. HSP27 alleviates cardiac aging in mice via a mechanism involving antioxidation and mitophagy activation. Oxidative medicine and cellular longevity, 2016, 2016.

Cite this article as: Li W, Liu F. Myocardial Cell Aging in the Elderly[J]. Aging Pathobiology and Therapeutics, 2020, 2(3): 134-142. 